\title{
RELATO DE CASO: RESPOSTA COMPLETA COM QUIMIOTERAPIA DE SEGUNDA LINHA EM CÂNCER DE BEXIGA ESTADIO IV
}

\author{
Carla Simone Moreira de FREITAS \& Paula Fontes Passalini GONÇALVES*
}

Hospital do Câncer de Muriaé, Fundação Cristiano Varella. Muriaé, Minas Gerais, Brasil.

*Autor para correspondência: paulafpassalini @ hotmail.com

DOI: http://dx.doi.org/10.18571/acbm.166

\section{RESUMO}

O câncer de bexiga é a neoplasia mais comum do sistema urinário, sendo raro ocorrer casos de resposta completa a quimioterapia em tumores avançados, quando não realizado cirurgia com intuito curativo. Objetivo: relatar o caso de um paciente que apresentou resposta completa da neoplasia vesical em estadio IV após quimioterapia de $2^{\mathrm{a}}$ linha.

Palavras chave: Sistema urinário; Câncer de bexiga; Quimioterapia.

\begin{abstract}
Bladder cancer is the most common neoplasm of the urinary system, and cases of complete response to chemotherapy in advanced tumors are rare, when surgery for curative purposes is not performed. Objective: To report the case of a patient who presented complete response of stage IV bladder neoplasia after $2^{\text {nd }}$ line chemotherapy.
\end{abstract}

Keywords: Urinary system; Bladder cancer; Chemotherapy.

\section{Introdução}

O câncer de bexiga é a neoplasia mais comum do sistema urinário, sendo o carcinoma urotelial/células transicionais o tipo histológico predominante em cerca de $90 \%$ dos casos (PLOEG, et al, 2009). O tratamento de pacientes em estadio IV é realizado com quimioterapia com regimes à base de Cisplatina. O quimioterápico inibidor de microtúbulo, Vinflunina, foi aprovado como primeira opção de regime de resgaste, após estudo de fase III publicado em 2010 pelo J Clin Oncol, que demonstrou aumento na sobrevida global de 2,3 meses em comparação com cuidados de suporte exclusivos, com taxa de resposta de 8,6\% e taxa de controle da doença de $41,1 \%$. Relatamos o caso de um paciente com carcinoma urotelial de bexiga em estadio IV, que apresentou resposta completa com uso de Vinflunina, após falha com Cisplatina e Gencitabina. Casos de resposta completa de neoplasias em estadio IV com quimioterapia de segunda linha são raros.

\section{Materiais e Métodos}

Foi realizada pesquisa bibliográfica para desenvolvimento do estudo. O relato de caso a ser descrito apresenta-se no campo da Saúde, área de Oncologia. A metodologia refere-se ao relato de caso, cujo paciente faz tratamento no Hospital do Câncer de Muriaé para neoplasia de bexiga, apresentando ausência de resposta com quimioterapia de primeira escolha, e resposta completa com quimioterapia de segunda linha. 


\section{Relato de Caso}

GDS, 73 anos, sexo masculino, ex-tabagista e etilista, história prévia de neoplasia gástrica tratada no ano de 2002 com gastrectomia total seguida de quimioterapia e radioterapia; sem outras doenças prévias.

Diagnóstico de carcinoma urotelial de bexiga com invasão da camada muscular e grau histológico 2, através de RTU (ressecção transuretral) de bexiga, devido hematúria e cistoscopia com lesão intra-vesical. Admitido no Hospital do Câncer de Muriaé com exames de estadiamento revelando estadio clinico IV (T4bNxM0). Ressonância Nuclear Magnética (RNM) do Abdome e Pelve demonstrava lesão de aspecto infiltrante, determinando espessamento da parede ânterolateral esquerda da bexiga. Esta lesão media 4,8 x 2,7 x 2,9 cm e acometia toda a espessura da parede vesical com extensão para a gordura perivesical adjacente, invasão de vasos ilíacos à esquerda e sem planos de clivagem com os ossos da pelve. Realizado quimioterapia com esquema padrão de Cisplatina com Gencitabina por 6 ciclos. Após término do tratamento paciente encontrava-se em estado geral regular e com hematúria macroscópica.

Tomografia Computadorizada (TC) de Abdome Total de reestadiamento após quimioterapia, demonstrava lesão de aspecto vegetante e infiltrante na parede ântero-lateral esquerda da bexiga, medindo 4,0 × 2,2 cm, envolvendo toda espessura da parede, projetando-se para a luz deste órgão, associada à discreta infiltração da gordura perivesical, conforme figuras 1 e 2 .

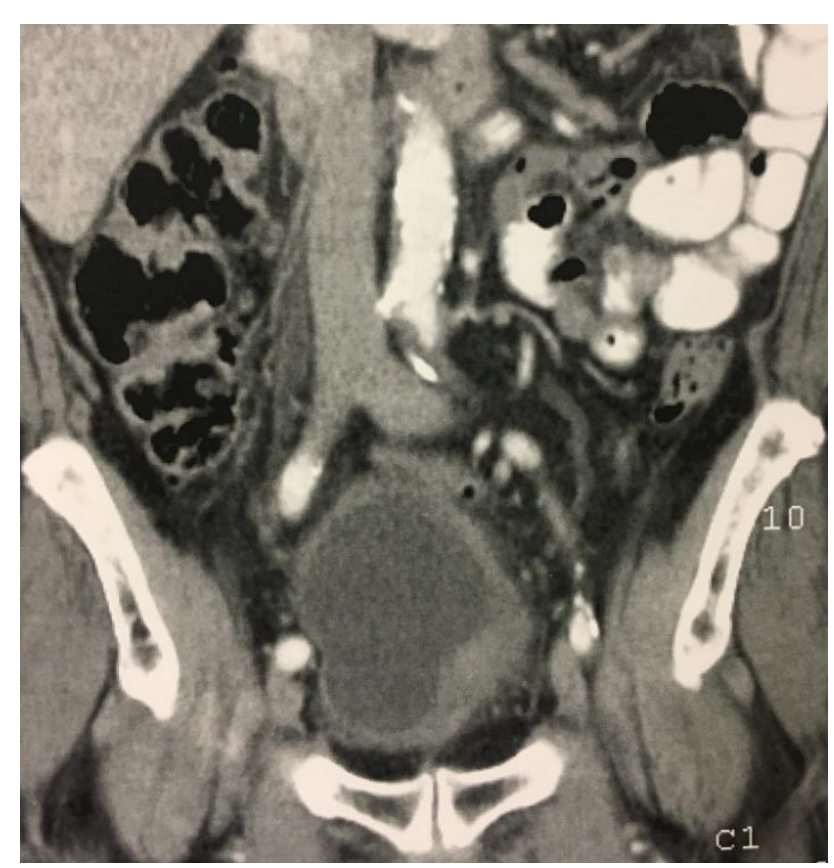

Figura 1: TC abdome (corte coronal) mostrando lesão vegetante e infiltrante na parede ântero-lateral esquerda da bexiga, medindo 4,0 x 2,2 cm, envolvendo toda espessura da parede, projetando-se para a luz, associada à discreta infiltração da gordura perivesical. 


\section{ACTA \\ Biomedica Brasiliensia}

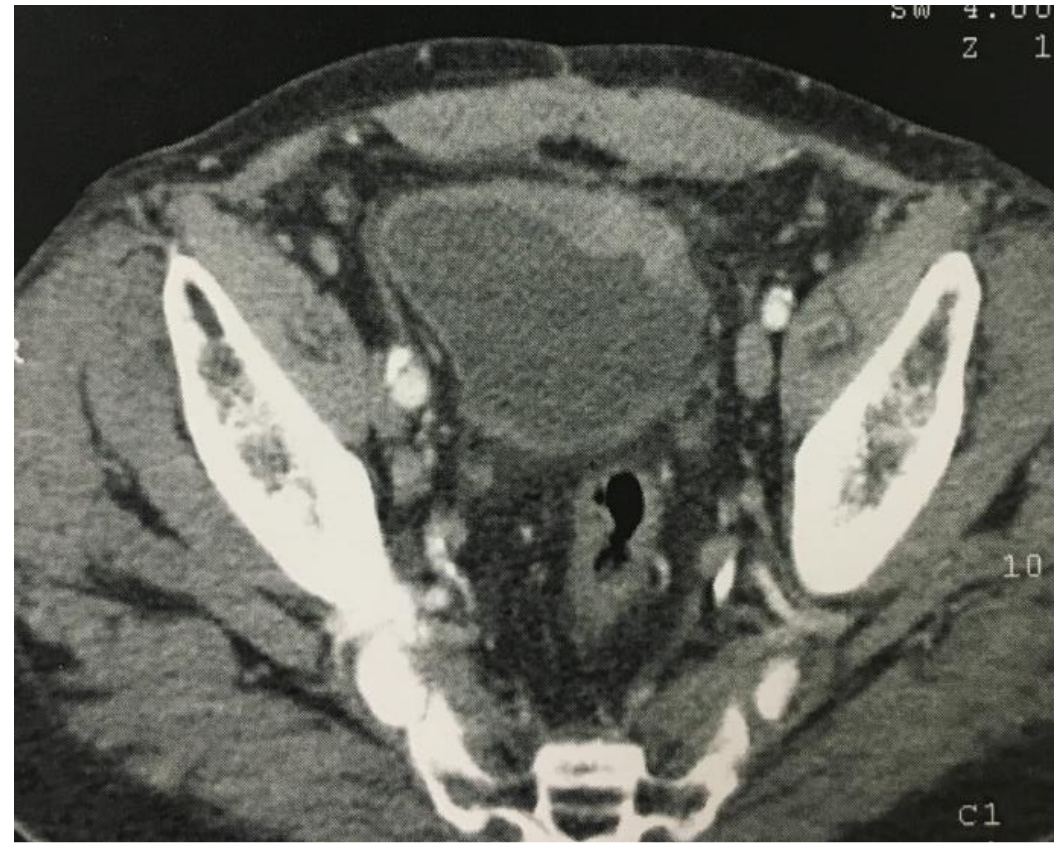

Figura 2: TC abdome (corte axial) mostrando lesão vegetante e infiltrante na parede ânterolateral esquerda da bexiga, medindo 4,0 × 2,2 cm, envolvendo toda espessura da parede, projetando-se para a luz, associada à discreta infiltração da gordura perivesical.

Foi iniciado quimioterapia de segunda linha com Vinflunina a cada 21 dias, por 6 ciclos. Após término do tratamento, paciente estava assintomático, com melhora no estado geral e sem novos episódios de hematúria. TC de Abdome e Pelve demonstrava espessamento difuso das paredes da bexiga, com irregularidade da região ântero-lateral esquerda, onde previamente existia a lesão vegetante e infiltrante, sem evidencia da lesão, conforme figuras 4 e 5 . Foi realizado cistoscopia que evidenciou bexiga com paredes espessadas e endurecidas, e ausência de lesões vegetantes. Demonstrando deste modo, resposta completa após tratamento com quimioterapia de segunda linha com Vinflunina.

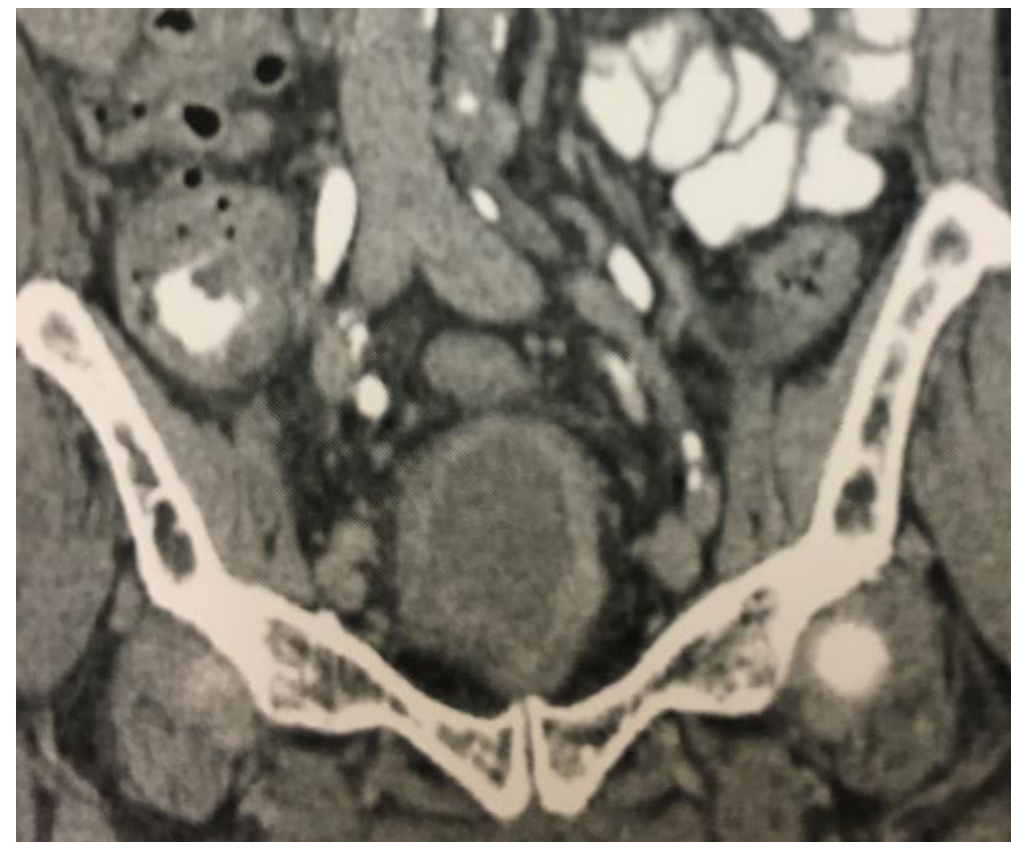

Figura 4: TC abdome (corte coronal) mostrando espessamento difuso das paredes da bexiga, com irregularidade da região ântero-lateral esquerda, sem evidencia de lesão. 


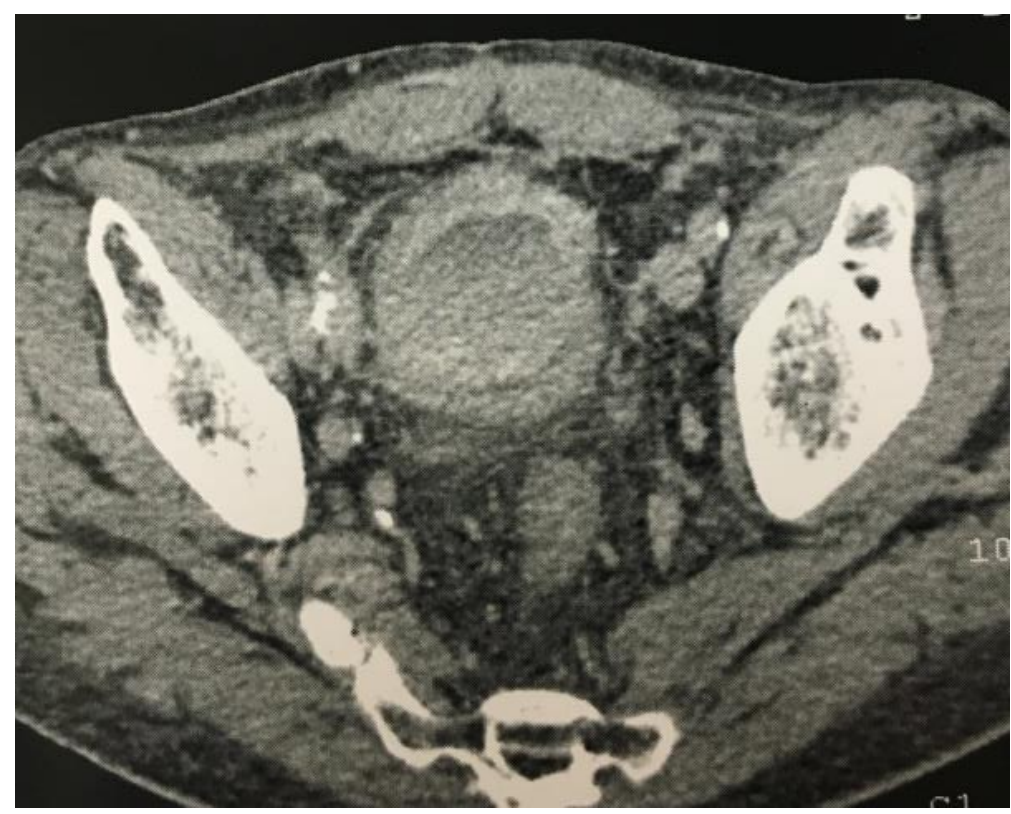

Figura 5: TC abdome (corte axial) mostrando espessamento difuso das paredes da bexiga, com irregularidade da região ântero-lateral esquerda, sem evidencia de lesão.

\section{Discussão}

Carcinoma de células transicionais é o tipo histológico predominante das neoplasias vesicais. A incidência aumenta com a idade, sendo 69 anos a média de idade ao diagnóstico em homens e 71 anos em mulheres (SCOSYREV, ET al, 2009). Entre os fatores de risco identificados, o mais importante é o tabagismo e exposições ocupacionais, como trabalhadores de indústrias de borracha, metal, couro e pintores (CUMBERBATCH, et al, 2016; KOGEVINAS, et al, 2003). A cistoscopia é o exame padrão para o diagnóstico da lesão; e o passo inicial para o estadiamento é a ressecção transuretral (RTU) do tumor vesical, para verificação do grau histológico e da profundidade de invasão, além de exames de imagem e laboratoriais. O estadiamento IV de acordo com a última edição do AJCC pode ser dividido em T4b (invasão de parede pélvica ou parede abdominal), ou linfonodos positivos, ou metástases a distância. O tratamento de pacientes em estadio 0 e I é a RTU seguida por instilação intravesical de BCG (bacilo de Calmette-Guérin) quando há critérios de risco para progressão/recidiva. Em estadios II a IV (M0) pode-se considerar quimioterapia neoadjuvante com esquemas a base de Cisplatina, seguido por cistectomia radical e linfadenectomia estendida. Se for identificada doença com invasão da camada muscular profunda é indicado quimioterapia adjuvante por 4 ciclos após cistectomia radical, podendo ser substituída por radioterapia ou máxima ressecção transuretral e quimioterapia paliativa nos pacientes não candidatos à cirurgia (SOLSONA, et al, 2010).

A primeira linha de quimioterapia adjuvante e para as doenças metastáticas são esquemas contendo Cisplatina. Geralmente a combinação de Cisplatina e Gencitabina é o protocolo padrão. Apesar de um significativo número de pacientes com doença em estadio IV apresentarem resposta objetiva durante terapia de $1^{\mathrm{a}}$ linha, a maioria terá progressão da doença (OK, et al, 2005). Foi aprovado o uso da Vinflunina como terapia de $2^{\mathrm{a}}$ linha em pacientes que falharam após uso de Cisplatina, em estudo de fase III publicado em 2010, que demonstrou benefício na sobrevida livre de progressão e na sobrevida global (BELLMUNT, et al, 2009). A Vinflunina é um alcalóide da vinca, inibidor dos microtúbulos, com boa tolerância pelos pacientes. As principais toxicidades dose-limitante incluem mucosite, constipação e neutropenia (BENNOUNA, et al, 2003). Regimes contendo Vinflunina estão sendo estudados também como terapia de $1^{\text {a }}$ linha em pacientes sem condições de receber Cisplatina (DE SANTIS, et al, 2013). 


\section{Conclusão}

Casos de neoplasia vesical em estadio T4b como o do caso relatado, sem possibilidades de se submeterem à cirurgia radical curativa, terem resposta completa com regimes quimioterápicos de segunda linha são considerados raros.

\section{Referências}

BELLMUNT J, THÉODORE C, DEMKOV T, et al. Phase III Trial of vinflunine plus Best supportive care compared with Best supportive care alone after a platinum-containing regimen in patients with advanced transitional cell carcinoma of the urothelial tract. J. Clin. Oncol. 27, 44544461 (2009).

BENNOUNA J, FUMOLEAU P, ARMAND J-P, et al. Phase I and pharmacokinecti study of the new vinca alkaloid vinflunine administered as a 10-min infusion every 3 weeks in patients with advenced solid tumors. Ann. Oncol. 14, 630-637 (2003).

CUMBERBATCH MG, ROTA M, CATTO JW, LA VECCHIA C. The Role of Tobacco Smoke in Bladder na Kidney Carcinogenesis: A Comparison of Exposures and Meta-analysis of Incidence and Mortality Risks. Eur Urol 2016; 70:458.

DE SANTIS M, WIECHNO P, LUCAS C, et al. Preliminary data on feasibility of vinfluninebased combinations as 1st line in CDDP-unfit patients with advanced urothelial carcinoma in a randomised international Phase II Trial (JASINT1). Eur. J. Cancer 49 (suppl. 2), S642 (2013).

KOGEVINAS M, 'T MANNETJE A, CORDIER S, et al. Occupation and bladder cancer among men in Western Europe. Cancer Causes Control 2003; 14:907.

OK JH, MEYERS F J, EVANS CP. Medical palliative care of patients with urological malignancies. J Urol 2005; 174:1177.

PLOEG M, ABEN KK, KIEMENEY LA. The present and future burden of urinary bladder cancer in the world. World J Urol 2009; 27:289.

SCOSYREV E, NOYES K, FENG C, MESSING E. Sex and racial differences in bladder cancer presentation and mortality in the US. Cancer 2009; 115:68.

SOLSONA E, IBORRA I, COLLADO A, et al. Feasibility of radical transurethral ressection as monotherapy for selected patients with muscle invasive bladder cancer. J Urol 2010; 184:475. 